\title{
Genetic variants of the matrix metalloproteinase family genes and risk for hypertension: a case-control study among northeastern Han Chinese
}

\author{
Yue $\mathrm{Qi}^{1,6}$, Hongye Zhao ${ }^{2,6}$, Yuefei Wang ${ }^{2,6}$, Yanli Wang ${ }^{3}$, Changzhu $\mathrm{Lu}^{2}$, Yu Xiao ${ }^{2}$, Bin Wang ${ }^{2}$ and \\ Wenquan $\mathrm{Niu}^{4,5}$
}

In this study, we sought to examine the association between 10 genetic variants in the matrix metalloproteinase (MMP) family genes and the risk of hypertension among northeastern Han Chinese. This was a hospital-based case-control study involving 1009 sporadic hypertensive patients and 756 age-, gender- and ethnicity-matched normotensive controls. The genotypes of the 10 examined variants were determined by PCR-ligase detection reaction method. The genotype/allele distributions of rs3025058 and rs679620 differed significantly between patients and controls, with a Bonferroni corrected $\alpha$ of 0.05/10. The probability of having hypertension was significant for rs 3025058 under the additive (odds ratio; $95 \%$ confidence interval; $P: 1.33 ; 1.16-1.53 ;<0.001)$ and dominant $(1.43 ; 1.18-1.73 ;<0.001)$ models and was significant for rs 679620 under the additive $(1.27 ; 1.1-1.46 ;<0.001)$ model after adjusting for confounders. In a combined analysis, when compared with the reference group (score $<3.5$ for unfavorable genotypes), participants in the medium- and high-risk groups had odds ratios that increased to $1.61(95 \% \mathrm{Cl}: 1.25-2.51 ; \boldsymbol{P}<0.001)$ and $1.92(95 \% \mathrm{Cl}: 1.54-2.39 ; \boldsymbol{P}<0.001)$ after adjustment, respectively. Interaction analysis showed that a three-locus model including rs3025058, rs679620 and rs243865 was the best, with a maximum testing accuracy of 0.6605 and a cross-validation consistency of $10(P=0.0022)$. Taken together, our findings suggest that the true association between individual variants and the risk of hypertension may not be revealed until combined analyses of multiple variants from genes involving a specific physiological or cellular function are performed. Moreover, we propose a three-locus model that can best characterize the genetic interactions of the MMP multiple gene family. Hypertension Research (2014) 37, 944-949; doi:10.1038/hr.2014.97; published online 15 May 2014

Keywords: gene; interaction; matrix metalloproteinase; variant

\section{INTRODUCTION}

The candidate-gene approach, which is hypothesis-driven and builds on a priori knowledge of the gene's biological functional impact on the trait or disease in question, has been extensively adopted in genetic association studies. ${ }^{1}$ However, one of the compelling problems facing most association studies is the lack of consistency in effect estimates. This can most likely be attributed to genetic heterogeneity across races/ethnicities, limited statistical power stemming from an insufficient sample size and lack of adjustment for potential confounders. Even in most studies where a significant association is reported, the odds ratios for individual genetic variants are often $<2$. $^{2,3}$ Such low-effect estimates are not surprising given that the pathogenesis of complex diseases such as hypertension usually involves a multistep, multigenic process. Furthermore, it is unlikely that any one individual genetic variant could explain a large proportion of variability in disease susceptibility. Therefore, tests for joint impacts of multiple candidate variants may provide valuable information in the search for hypertension-susceptibility genes.

For this project, we focused on the components of the matrix metalloproteinase (MMP) family as hypertension candidate genes. MMPs are zinc-dependent endopeptidases that belong to a large family of structurally related proteases capable of degrading all types of extracellular matrix proteins. ${ }^{4}$ Extracellular matrix proteins have a supporting and structural role in the vascular wall, and their high content has been found to be associated with an increased risk of hypertension and target organ damage. ${ }^{5}$ MMPs can also modify extracellular matrix proteins and exert a significant impact on many physiological and pathological processes. ${ }^{5,6}$ Consequently, the genes

${ }^{1}$ Department of Epidemiology, Beijing An Zhen Hospital, Capital Medical University, Beijing Institute of Heart, Lung and Blood Vessel Diseases, Beijing, China; ${ }^{2}$ Department of Physiology, Qiqihar Medical University, Qiqihar, Heilongjiang, China; ${ }^{3}$ The Third Division of Cardiology, Department of Internal Medicine, The Second Affiliated Hospital of Qiqihar Medical University, Qiqihar, Heilongiiang, China; ${ }^{4}$ State Key Laboratory of Medical Genomics, Shanghai Jiao Tong University School of Medicine, Shanghai, China and ${ }^{5}$ Shanghai Institute of Hypertension, Ruijin Hospital, Shanghai Jiao Tong University School of Medicine, Shanghai, China

6These authors contributed equally to this work.

Correspondence: Dr Y Qi or Dr B Wang or Dr W Niu, Department of Epidemiology, Beijing An Zhen Hospital, Capital Medical University, Beijing Institute of Heart, Lung and Blood Vessel Diseases, No. 2 An Zhen Road, Chaoyang District, Beijing 100029, China.

E-mail: qiyue_bjcn@163.com or wangbinqqhr@163.com or niuwenquan_shcn@163.com

Received 13 January 2014; revised 4 March 2014; accepted 5 April 2014; published online 15 May 2014 
encoding MMPs are regarded as logical candidates for determining the molecular mechanisms underlying hypertension. MMPs are classified into collagenases (MMP-1, -8, -13, -18), stromelysins (MMP-3, -10, -11), gelatinases (MMP-2, -9) and membrane-type MMPs. ${ }^{7}$ Each MMP family member is coded by a separate gene and has a different tissue distribution and bioactive role. ${ }^{8}$ The regulatory expression levels of MMP genes may involve mRNA stability mechanisms in the cytoplasm. Genetic defects that have been reported to affect the binding site of transcription factors or cause the loss of function of MMP enzymes may also be involved. ${ }^{9-11}$ Considering the polymorphic nature of the encoding genes of MMP family members, it is of added interest to determine which MMP genetic variants have the functional potential to affect the final bioavailability of the family members and thus the progression of hypertension.

To generate more information, we sought to examine the association between a comprehensive panel of genetic variants in the MMP family genes and the risk of hypertension in a large northeastern Han Chinese population. In total, 10 genetic variants were selected from five genes encoding MMP-1 (rs1799750, rs1144393), MMP-2 (rs243865, rs243864), MMP-3 (rs3025058, rs617819, rs679620), MMP-9 (rs3918242, rs2664538) and MMP-12 (rs2276109). All study variants have previously been examined in published association studies and are located in the promoter or coding regions, with minor allele frequencies of at least $5 \% .{ }^{12-16}$ In the present study, we examined the combined effects of risk alleles of all the examined variants and evaluated their high-order gene-to-gene interactions.

\section{METHODS}

\section{Study design}

This was a hospital-based case-control study, as we have previously reported. ${ }^{17-19}$

\section{Study population}

All study participants were recruited from Qiqihar city, Heilongjiang province, China. The study was approved by the institutional review board of Qiqihar Medical University, and written informed consent was obtained from each participant at the time of enrollment. This study was performed in agreement with the guidelines outlined in the Declaration of Helsinki.

Blood pressure was measured using a calibrated mercury sphygmomanometer (Baumanometer, W.A. Baum Co, Inc., Copiague, NY, USA) with an appropriate adult cuff size by certified examiners. Hypertension was defined as a mean systolic blood pressure $>140 \mathrm{~mm} \mathrm{Hg}$ or a diastolic blood pressure $>90 \mathrm{~mm} \mathrm{Hg}$ or the current use of antihypertensive drugs. As recommended by Tobin et al. ${ }^{20}$ for participants taking antihypertensive medication, blood pressure was determined by adding 15 and $10 \mathrm{~mm} \mathrm{Hg}$ to the systolic and diastolic blood pressure, respectively.

All study participants were assigned to the hypertensive group or normotensive group based on the results of clinical and laboratory examinations. The hypertensive group consisted of inpatients or outpatients of the Second Affiliated Hospital of Qiqihar Medical University. Hypertensive patients with clinical evidence of secondary hypertension and renal disease were excluded. The normotensive controls, who underwent a medical examination at the same hospital, were clinically confirmed to be free of hypertension and had a negative family history of hypertension in their first-degree relatives. All study participants were genetically unrelated local residents of Han Chinese descent who were recruited consecutively between June 2008 and December 2012.

\section{Sample size}

The hypertensive group included 1009 sporadic patients with a mean age of 64.48 (s.d.: 8.53 ) years. Men comprised $54.31 \%$ of this study population. The remaining participants $(n=756)$ whose blood pressure was normal formed the age-, gender- and ethnicity-matched control group.

\section{Measurement}

Age, gender, body weight and height were recorded at the time of enrollment. For hypertensive patients, age referred to the age of first onset of hypertension. Body weight was measured in light clothing and bare feet. Body mass index (BMI) was calculated as weight in kilograms divided by height in meters squared. Blood samples were collected after overnight fasting, and the sera were isolated immediately by centrifugation. The plasma levels of triglycerides, total cholesterol, high-density lipoprotein cholesterol, blood urea nitrogen, creatinine and urea acid were determined enzymatically using the available kits and auto-analyzers. High-sensitivity C-reactive protein was measured using a particle-enhanced immunoturbidimetric method.

\section{Genotyping}

Genomic DNA was extracted from peripheral blood leukocytes by a standard phenol-chloroform method and stored at $-40{ }^{\circ} \mathrm{C}$ until required for batch genotyping. The genotypes of the 10 examined variants were determined by the PCR-ligase detection reaction (PCR-LDR) method as previously described. $^{21}$

PCR reactions were conducted in an EDC-810 Amplifier (Dongsheng Innovation Biotech, Beijing, China). The cycling parameters were as follows: $94^{\circ} \mathrm{C}$ for $2 \mathrm{~min} ; 35 \mathrm{cycles}$ of $94^{\circ} \mathrm{C}$ for $20 \mathrm{~s}, 60^{\circ} \mathrm{C}$ for $20 \mathrm{~s}$ and $72^{\circ} \mathrm{C}$ for $20 \mathrm{~s}$; and a final extension step at $72^{\circ} \mathrm{C}$ for $3 \mathrm{~min}$.

For each variant, two specific probes were synthesized to discriminate specific bases, with one common probe labeled with 6-carboxy-fluorescein at the $3^{\prime}$ end and phosphorylated at the $5^{\prime}$ end. The multiplex ligation reaction was conducted in a reaction volume of $10 \mu \mathrm{l}$ containing $2 \mu \mathrm{l}$ of PCR product, $1 \mu \mathrm{l}$ of $10 \times$ Taq DNA ligase buffer, $1 \mu \mathrm{M}$ of each discriminating probe and $5 \mathrm{U}$ of Taq DNA ligase. The ligation parameters were 30 cycles of $94^{\circ} \mathrm{C}$ for $30 \mathrm{~s}$ and $56^{\circ} \mathrm{C}$ for $3 \mathrm{~min}$. After the reaction, $1 \mu \mathrm{l}$ of LDR reaction product was mixed with $1 \mu \mathrm{l}$ of ROX passive reference and $1 \mu \mathrm{l}$ of loading buffer before being denatured at $95^{\circ} \mathrm{C}$ for $3 \mathrm{~min}$ and chilled rapidly in ice water. The fluorescent products of the LDR were differentiated using an ABI 3730XL sequencer (Applied Biosystems, Foster City, CA, USA).

To test the accuracy of this PCR-LDR method, 96 DNA samples were randomly selected and run in duplicates with $100 \%$ concordance.

\section{Statistical analysis}

The Student's $t$-test was used to test for differences between patients and controls for quantitative variables. The $\chi^{2}$ test was used to assess the goodnessof-fit of the observed allele frequencies with the expected frequencies according to the Hardy-Weinberg equilibrium and the genotype/allele frequencies between the two groups. Statistical power was estimated by the PS (Power and Sample Size Calculations) software (version 3.0, Department of Biostatistics, Vanderbilt University, Nashville, TN, USA).

Given that no definitive previous associations between all examined variants and hypertension risk were available after reviewing the literature, we primarily treated the minor-variant allele at each locus as the 'risk' allele. Each genotype of the 10 examined variants was regressed in an unconditional logistic regression model under the assumptions of additive (major homozygotes $v s$. heterozygotes vs. minor homozygotes), dominant (major homozygotes vs. heterozygotes plus minor homozygotes) and recessive (major homozygotes plus heterozygotes $v s$. minor homozygotes) models of inheritance after controlling for age, gender and BMI. Risk estimates are expressed as the odds ratio (OR) and its $95 \%$ confidence interval (95\% CI). If the OR was less than unity for the minor-variant allele, the alternative major allele was reassigned as the risk allele. The best-fitting model among three assumed genetic models was the one with the smallest adjusted $P$-value. In cases where the genotype count for the homozygous variant genotype was less than five in both patients and controls, only the dominant model with the highest statistical power was considered.

The cumulative effects of multiple variants in the MMP family genes on hypertension risk were assessed by an unfavorable genotype analysis using six variants (rs1144393, rs243865, rs3025058, rs679620, rs2664538 and rs2276109) that exhibited a borderline significant association in a single-locus analysis $(P$ for the best-fitting model $<0.10)$. The unfavorable genotype referred to the genotype carrying the risk allele(s). The best-fitting model was the additive 
model for rs1144393 (unfavorable genotype: WW and WM), rs243865 (WW and WM) and rs679620 ( $\mathrm{MM}$ and $\mathrm{WM}$ ), the dominant model for rs3025058 (WW + WM) and rs2276109 (WW) and the recessive model for rs2664538 (WW + WM), where WW is homozygous wild types, WM is heterozygotes and MM is homozygous variants. In the additive model, an unfavorable genotype carrying one and two risk alleles was assigned a score of 0.5 and 1 , respectively, and in the other two models, an unfavorable genotype was assigned a score of 1 . The number of unfavorable genotypes was summed for each participant. The statistical analyses described above were completed using the STATA software (version 11.2, StataCorp, College Station, TX, USA) in Windows. A two-tailed $P<0.05$ was considered statistically significant.

The potential interactions of multiple variants in the MMP family genes were identified and characterized by using an open-source data-mining

Table 1 Baseline characteristics of all the study subjects

\begin{tabular}{|c|c|c|c|}
\hline Characteristics & Patients $(\mathrm{n}=1009)$ & Controls $(n=756)$ & P-value \\
\hline Age (years) & $64.48 \pm 8.53$ & $64.23 \pm 10.13$ & 0.751 \\
\hline Gender (males) & $54.31 \%$ & $53.84 \%$ & 0.843 \\
\hline $\mathrm{BMI}\left(\mathrm{kg} \mathrm{m}^{-2}\right)$ & $27.89 \pm 6.29$ & $23.18 \pm 3.77$ & $<0.001$ \\
\hline Antihypertensive treatment & $14.17 \%$ & $0 \%$ & $<0.001$ \\
\hline Adjusted SBP $(\mathrm{mm} \mathrm{Hg})$ & $147.31 \pm 16.52$ & $109.76 \pm 17.97$ & $<0.001$ \\
\hline Adjusted DBP (mm Hg) & $89.09 \pm 15.92$ & $71.37 \pm 11.43$ & $<0.001$ \\
\hline Fasting glucose $\left(\mathrm{mmoll}^{-1}\right)$ & $6.14 \pm 2.15$ & $5.33 \pm 1.12$ & $<0.001$ \\
\hline $\mathrm{TG}\left(\mathrm{mmol} \mathrm{I}^{-1}\right)$ & $1.90 \pm 1.04$ & $1.77 \pm 0.95$ & 0.024 \\
\hline $\mathrm{TC}\left(\mathrm{mmoll} \mathrm{I}^{-1}\right)$ & $4.59 \pm 1.18$ & $4.59 \pm 0.91$ & 0.995 \\
\hline HDL-C (mmoll-1) & $1.12 \pm 0.32$ & $1.24 \pm 0.34$ & $<0.001$ \\
\hline BUN (mmoll-1) & $5.92 \pm 3.88$ & $5.79 \pm 4.35$ & 0.557 \\
\hline Creatinine $\left(\mu \mathrm{moll} \mathrm{I}^{-1}\right)$ & $87.47 \pm 36.79$ & $81.80 \pm 25.13$ & 0.002 \\
\hline Uric acid $\left(\mu \mathrm{mol} \mathrm{I}^{-1}\right)$ & $329.12 \pm 100.31$ & $333.98 \pm 95.54$ & 0.374 \\
\hline $\operatorname{hsCRP}\left(\mathrm{mmoll}^{-1}\right)$ & $12.37 \pm 41.42$ & $2.21 \pm 3.71$ & 0.001 \\
\hline
\end{tabular}

Abbreviations: BMI, body mass index; BUN, blood urea nitrogen; DBP, diastolic blood pressure; HDL-C, high-density lipoprotein cholesterol; hsCRP, high-sensitivity C-reactive protein; SBP, systolic blood pressure, TC, total cholesterol; TG, triglyceride.

${ }^{a} P$-values were computed by unpaired $t$-test or Mann-Whitney $U$ test for quantitative variables and by $\chi^{2}$ test for qualitative variables. multifactor dimensionality reduction (MDR) approach (version 3.0, available at the website www.epistasis.org). ${ }^{22,23}$ This approach aims to construct all possible combinations of the above six variants. The accuracy of each MDR model is evaluated by a Bayes classifier in the context of a 10 -fold crossvalidation. A single best model simultaneously has the maximum testing accuracy and cross-validation consistency (a measure of the number of times of 10 divisions of the data set that the best model is extracted). Statistical significance was evaluated using a 1000 -fold permutation test to compare observed testing accuracies with those expected under the null hypothesis of null association. The permutation test corrects for multiple testing by repeating the entire analyses on 1000 data sets that are consistent with the null hypothesis.

\section{RESULTS}

Baseline characteristics

Demographic and clinical variables are compared between hypertensive patients and normotentive controls in Table 1. The distribution of age $(P=0.751)$ and gender $(P=0.843)$ did not significantly differ between patients and controls. The levels of BMI $(P<0.001)$, systolic and diastolic blood pressure (both $P<0.001$ ), fasting glucose $(P<0.001)$, triglycerides $(P=0.024)$, creatinine $(P=0.002)$ and high-sensitivity $\mathrm{C}$-reactive protein were significantly higher, whereas the high-density lipoprotein cholesterol level was significantly lower in patients than in controls $(P<0.001$ for all). The proportion of patients undergoing antihypertensive treatment was $14.17 \%$.

\section{Single-locus analysis}

Table 2 shows the genotype distributions and allele frequencies of the 10 examined variants in the MMP family genes between hypertensive patients and normotensive controls. No deviations from the HardyWeinberg equilibrium were observed in normotensive controls for all the 10 variants examined $(P>0.05)$. The genotype and allele distributions of rs243865, rs3025058, rs679620 and rs2276109 differed significantly between patients and controls at the significant level of 0.05, whereas with a Bonferroni corrected $\alpha$ of $0.05 / 10$ (here, 10 is the

Table 2 Genotype distributions and allele frequencies of the 10 examined variants in the 5 MMP family genes between hypertensive patients and normotensive controls

\begin{tabular}{|c|c|c|c|c|c|c|c|c|}
\hline Gene: variant & $W / M$ & Class & $W W$ & $W M$ & $M M$ & $\mathrm{P}$-value & $M(\%)$ & P-value \\
\hline \multirow[t]{2}{*}{ MMP-1: rs1799750 } & $\mathrm{GG} / \mathrm{G}$ & Patients & 351 & 481 & 177 & 0.613 & 41.38 & 0.357 \\
\hline & & Controls & 253 & 357 & 146 & & 42.92 & \\
\hline \multirow[t]{2}{*}{ MMP-1: rs1144393 } & $A / G$ & Patients & 381 & 499 & 129 & 0.146 & 37.51 & 0.056 \\
\hline & & Controls & 256 & 385 & 115 & & 40.67 & \\
\hline \multirow[t]{2}{*}{ MMP-2: rs243865 } & $\mathrm{C} / \mathrm{T}$ & Patients & 580 & 357 & 72 & 0.044 & 24.83 & 0.011 \\
\hline & & Controls & 391 & 297 & 68 & & 28.64 & \\
\hline \multirow[t]{2}{*}{ MMP-2: rs243864 } & $\mathrm{T} / \mathrm{G}$ & Patients & 887 & 118 & 4 & 0.888 & 6.24 & 0.974 \\
\hline & & Controls & 664 & 90 & 2 & & 6.22 & \\
\hline \multirow[t]{2}{*}{ MMP-3: rs3025058 } & $6 \mathrm{~A} / 5 \mathrm{~A}$ & Patients & 394 & 465 & 150 & $<0.001$ & 37.91 & $<0.001$ \\
\hline & & Controls & 361 & 317 & 78 & & 31.28 & \\
\hline \multirow[t]{2}{*}{ MMP-3: rs617819 } & $C / G$ & Patients & 439 & 432 & 138 & 0.273 & 35.08 & 0.212 \\
\hline & & Controls & 340 & 332 & 84 & & 33.07 & \\
\hline \multirow[t]{2}{*}{ MMP-3: rs679620 } & $\mathrm{G} / \mathrm{A}$ & Patients & 368 & 488 & 153 & 0.004 & 39.35 & $<0.001$ \\
\hline & & Controls & 324 & 351 & 81 & & 33.93 & \\
\hline \multirow[t]{2}{*}{ MMP-9: rs3918242 } & $\mathrm{C} / \mathrm{T}$ & Patients & 725 & 260 & 24 & 0.536 & 15.26 & 0.862 \\
\hline & & Controls & 545 & 186 & 24 & & 15.50 & \\
\hline \multirow[t]{2}{*}{ MMP-9: rs2664538 } & $A / G$ & Patients & 427 & 462 & 120 & 0.086 & 34.79 & 0.043 \\
\hline & & Controls & 296 & 344 & 116 & & 38.10 & \\
\hline \multirow[t]{2}{*}{ MMP-12: rs2276109 } & $A / G$ & Patients & 708 & 281 & 20 & 0.018 & 15.91 & 0.006 \\
\hline & & Controls & 483 & 252 & 21 & & 19.44 & \\
\hline
\end{tabular}

Abbreviations: MMP, matrix metalloproteinase; W/M, wild allele/mutant allele.

$P$ values were computed by using $\chi^{2}$ test based on the $3 \times 2$ contingency tables for genotype comparisons and on the $2 \times 2$ contingency tables for allele comparisons. 
Table 3 Risk prediction of the 10 examined variants in the 5 MMP family genes for hypertension risk under three genetic models of inheritance

\begin{tabular}{lccc}
\hline SNP & Additive model & Dominant model & Recessive model \\
\hline rs1799750 & $0.94 ; 0.82-1.07 ; 0.363$ & $0.94 ; 0.77-1.15 ; 0.563$ & $0.89 ; 0.7-1.13 ; 0.341$ \\
rs1144393 & $0.87 ; 0.75-1.0 ; 0.05$ & $0.84 ; 0.69-1.03 ; 0.092$ & $0.82 ; 0.62-1.07 ; 0.144$ \\
rs243865 & $0.83 ; 0.72-0.96 ; 0.013$ & $0.79 ; 0.66-0.96 ; 0.016$ & $0.78 ; 0.55-1.1 ; 0.154$ \\
rs243864 & $1.01 ; 0.76-1.33 ; 0.974$ & $0.99 ; 0.74-1.33 ; 0.96$ & $1.5 ; 0.27-8.21 ; 0.64$ \\
rs3025058 & $1.33 ; 1.16-1.53 ;<0.001$ & $1.43 ; 1.18-1.73 ;<0.001$ & $1.52 ; 1.13-2.03 ; 0.005$ \\
rs617819 & $1.09 ; 0.95-1.25 ; 0.221$ & $1.06 ; 0.88-1.28 ; 0.54$ & $1.27 ; 0.95-1.69 ; 0.108$ \\
rs679620 & $1.27 ; 1.1-1.46 ;<0.001$ & $1.31 ; 1.08-1.58 ; 0.007$ & $1.49 ; 1.12-1.99 ; 0.007$ \\
rs3918242 & $0.98 ; 0.82-1.18 ; 0.864$ & $1.02 ; 0.83-1.26 ; 0.864$ & $0.74 ; 0.42-1.32 ; 0.311$ \\
rs2664538 & $0.87 ; 0.76-1.0 ; 0.044$ & $0.88 ; 0.72-1.06 ; 0.181$ & $0.74 ; 0.57-0.98 ; 0.035$ \\
rs2276109 & $0.77 ; 0.65-0.93 ; 0.005$ & $0.75 ; 0.62-0.92 ; 0.005$ & $0.71 ; 0.38-1.32 ; 0.274$ \\
\hline
\end{tabular}

Abbreviations: MMP, matrix metalloproteinase; SNP, single-nucleotide polymorphism.

Data are expressed as odds ratio; $95 \%$ confidence interval; $P$-values. $P$ was calculated after controlling for age, gender and body mass index.

Table 4 Combined effect analysis by the number of unfavorable genotypes from six significant variants and hypertension risk ${ }^{a}$

\begin{tabular}{lccc}
\hline Unfavorable genotype & Patients & Controls & OR; 95\% Cl; P-value \\
\hline Low risk (score $\leqslant 3.5)$ & 382 & 397 & 1 (reference group) \\
Medium risk (score: $(3.5-4))$ & 223 & 142 & $1.61 ; 1.25-2.51 ;<0.001$ \\
High risk (score $>4)$ & 404 & 217 & $1.92 ; 1.54-2.39 ;<0.001$
\end{tabular}

Abbreviations: OR, odds ratio; 95\% $\mathrm{Cl}, 95 \%$ confidence interval.

aThe six variants included are rs1144393, rs243865, rs3025058, rs679620, rs2664538 and rs2276109.

total number of examined variants), only rs3025058 ( $P$ for genotype and allele: $<0.001$ and $<0.001$, respectively) and rs679620 ( $P$ for genotype and allele: 0.004 and $<0.001$, respectively) differed significantly between the two groups. The power to reject the null hypothesis of no differences in risk allele frequencies of rs3025058 and rs679620 between the two groups was $98.4 \%$ and $91.1 \%$, respectively.

The risk prediction of the 10 examined variants for hypertension risk under the three genetic models is presented in Table 3. After the Bonferroni's correction for multiple tests (Bonferroni's significance threshold $P=0.05 / 10$ ), the odds of having hypertension was significant for $\mathrm{rs} 3025058$ under the additive $(\mathrm{OR}=1.33$; 95\% CI: $1.16-1.53 ; P<0.001)$ and dominant $(\mathrm{OR}=1.43$; 95\% CI: $1.18-1.73$; $P<0.001)$ models and was significant for rs679620 under the additive ( $\mathrm{OR}=1.27$; 95\% CI: 1.1-1.46; $P<0.001)$ model after controlling for age, gender and BMI. Risk estimates were marginally significant for rs3025058 under the recessive $(\mathrm{OR}=1.52 ; 95 \%$ CI: $1.13-2.03$; $P=0.005)$ model and for $r 2276109$ under the additive $(\mathrm{OR}=0.77$; 95\% CI: $0.65-0.93 ; P=0.005)$ and dominant $(\mathrm{OR}=0.75 ; 95 \% \mathrm{CI}$ : $0.62-0.92 ; P=0.005)$ models. No significance was attained for the association of the other variants with hypertension.

\section{Cumulative effect analysis}

To examine whether the effects of multiple variants in the MMP family genes were additive on hypertension risk, a cumulative analysis of unfavorable genotypes of significant variants was performed (Table 4). Given our sample size, we trichotomized each participant into the low-, medium- and high-risk groups based on the tertile distribution of the total risk score of unfavorable genotypes in controls, and we set participants with a score of $\leqslant 3.5$ as the reference group. Overall, there was a trend towards an increased risk of hypertension with increasing numbers of unfavorable genotypes. Compared with the reference group, participants with scores spanning
$(3.5,4)$ and $>4$ had ORs that increased to 1.61 (95\% CI: $1.25-2.51$; $P<0.001)$ and $1.92(95 \% \mathrm{CI}: 1.54-2.39 ; P<0.001)$ after controlling for age, gender and BMI, respectively. Moreover, each additional increase in risk score was significantly associated with a $44 \%$ increase in hypertension risk (95\% CI: 1.29-1.6; $P<0.001)$ after adjustment.

\section{Genetic interaction analysis}

To further explore the potential interaction of six significant variants, we used the promising data-mining analytical approach MDR. As summarized in Table 5, each best model across all possible combinations is assessed by the testing accuracy, cross-validation consistency and significance level. Out of the six derived MDR models, a threelocus model including rs3025058, rs679620 and rs243865 was chosen as the overall best model with the maximum testing accuracy of 0.6605 and cross-validation consistency of 10 . This best model was significant at $P=0.0022$, suggesting that a model this good or better was observed less than 3 out of the 1000 permutations and thus was unlikely to hinge on the null hypothesis of null association.

\section{DISCUSSION}

In this study, we systematically evaluated the individual and joint effects of the 10 genetic variants in the 5 MMP family genes on the risk of hypertension in a large northeastern Han Chinese population. The most noteworthy finding was that the true association between individual variants and hypertension risk may not be revealed until the combined analyses of multiple variants from genes involving a specific physiological or cellular function are performed. Moreover, we proposed a three-locus model that can best characterize genetic interactions of multiple variants in the MMP family genes. Our results not only highlight the complexity of the joint effects of genetic variation on disease risk but also provide evidence supporting the logical functional role of the MMP family genes in the pathogenesis of hypertension.

It is well known that hypertension is an established independent risk factor for cardiovascular disease, which is characterized by an increase in extracellular matrix content. ${ }^{24} \mathrm{~A}$ substantial body of evidence supports a close relationship between high extracellular matrix content and hypertension. ${ }^{5,25}$ It is reasonable to expect that the degradation of extracellular matrix proteins by MMPs may contribute to the pathogenesis of cardiovascular disease as well as hypertension. Recently, Schmid-Schonbein ${ }^{6}$ has written an excellent editorial commentary on the emerging opportunities for MMPs in the optimal management of hypertension and its complications. 
Table 5 Summary of MDR analysis on six significant variants in the five MMP family genes

\begin{tabular}{lrr}
\hline Best combination of each model & Testing accuracy & Cross-validation consistency \\
\hline rs3025058 & 0.6038 & 6 \\
rs3025058, rs679620 & 0.6128 & 8 \\
rs3025058, rs679620, rs243865 & 0.6605 & 0.1197 \\
rs3025058, rs679620, rs243865, rs1144393 & 0.6167 & 0.0783 \\
rs3025058, rs679620, rs243865, rs1144393, rs2664538 & 0.5931 & $0.0022^{a}$ \\
rs3025058, rs679620, rs243865, rs1144393, rs2664538, rs2276109 & 0.6025 & 0.0385 \\
\hline
\end{tabular}

Abbreviations: MDR, multifactor dimensionality reduction; MMP, matrix metalloproteinase.

aThe overall best model.

Therefore, understanding the genetic and/or environmental determinants of MMP activation should be a high priority.

Assessing the association between the MMP family genes and cardiovascular disease has been widely used to identify the regions of the genome and candidate variants that contribute to disease susceptibility. However, these associations have always been debated because of inconsistent reports. Recently, we have undertaken a systematic review of the literature by means of a meta-analysis on the association of the MMP family multiple variants with coronary artery disease, and we found strong evidence of a disease susceptibility for the MMP-3 and MMP-9 genes. ${ }^{12}$ In the present study, we confirmed the positive association of two functional variants of the MMP-3 gene with hypertension risk in a single-locus analysis, reinforcing an independent leading role of the MMP-3 gene in the development of cardiovascular disease. Moreover, our interaction analysis identified another promoter variant rs243865 in the MMP-2 gene that significantly interacted with the MMP-3 gene in the best prediction of hypertension. It is of interest to note that rs 243865 showed no statistical association with hypertension in our single-locus analysis, suggesting that there is evidence of epistasis, that is, the effect of one variant may not be disclosed if the effect of another locus is not considered. ${ }^{26}$ Although our data failed to detect a relationship between MMP-9 genetic variants and hypertension risk, there was evidence that MMP-2 and MMP-9 levels were inversely correlated with arterial stiffness in healthy participants. ${ }^{27}$ Furthermore, blockade of MMP-9 activity resulted in an increase in pulse pressure. ${ }^{28} \mathrm{We}$ cannot therefore exclude the possibility that a causal variant exists in the MMP-9 gene that is not recognized by the markers selected in association with hypertension or other specific forms of cardiovascular disease. Fully addressing this possibility will require a more comprehensive genetic approach, such as the selection of tagging variants in most key genes that are involved in a specific physiological or cellular function. This approach would offer more convincing evidence for the relevance of the examined genes in disease risk.

In many cases, genetic association studies are complicated by genetic heterogeneity and insufficient study power. On the one hand, genetic heterogeneity is an inevitable problem in disease identification strategies, ${ }^{29}$ and it may explain in part the inconsistent associations found across ethnic groups. One potential strategy to decrease genetic heterogeneity is to use homogeneous populations. ${ }^{30}$ In fact, our study population is characterized by genetic homogeneity and geographic stability. In addition, the subjects are most likely uniform in terms of environmental exposures, including habitual dietary intake of high amount of salt and $\mathrm{fat}^{31}$ and a lower rate of hypertension recognition and treatment. These factors render this population more appropriate for enhancing our understanding of the genetic predisposition to essential hypertension. Conversely, as recommended by Cardon and
Bell $^{32}$, recruiting $\geqslant 1000$ individuals in each group is required to yield a firm conclusion. Although our sample size of 1009 hypertensive patients and 756 normotensive controls fails to meet this requirement, an $a$ priori power calculation suggested that we had $>90 \%$ power to detect the significant genetic variants of realistic effect sizes.

Several limitations should be considered when interpreting our results. First, because this was a retrospective case-control study, our data cannot prove the existence of a cause-effect relationship between examined genetic variants and hypertension. ${ }^{33}$ Second, this study, which involved 1765 subjects, may be underpowered to demonstrate small risk effects. Third, the fact that only 10 common variants were selected from the promoter or coding regions of the 5 MMP family genes leaves an opening for the incorporation of more functional variants, especially the low-penetrance variants from the MMP family genes. Fourth, data on the types of antihypertensive drugs used and the treatment period were not available, precluding further analyses of drug effects on our findings. Fifth, the MDR method used in this study has some underlying drawbacks, including computational intensiveness, indistinct interpretation, lack of sensitivity and heterogeneity-free assumption. ${ }^{34,35}$ Finally, the fact that our study participants were of northeastern Han Chinese descent limits the generalizability of our findings and necessitates further confirmation in other ethnic populations.

In summary, our findings suggest that the true association between individual variants and the risk of hypertension may not be revealed until combined analyses of multiple variants from genes involving a specific physiological or cellular function are performed. Nevertheless, for practical reasons, we hope that this study will serve to encourage more comprehensive evaluation of a larger panel of genetic variants in the MMP family genes in other independent groups, thus furthering our understanding of the genetic underpinnings of hypertension and related cardiovascular diseases.

\section{CONFLICT OF INTEREST}

The authors declare no conflict of interest.

\section{ACKNOWLEDGEMENTS}

Funding sources: This study was supported by grants from the Beijing New Star Program (Z111107054511072), the National Natural Science Foundation of China (81000109) and the Department of Education Science and Technology Research Project in Heilongiiang Province (12521621).

1 Wu X, Gu J, Grossman HB, Amos Cl, Etzel C, Huang M, Zhang Q, Millikan RE, Lerner S, Dinney CP, Spitz MR. Bladder cancer predisposition: a multigenic approach to DNA-repair and cell-cycle-control genes. Am J Hum Genet 2006; 78: 464-479.

2 Neumann AS, Sturgis EM, Wei Q. Nucleotide excision repair as a marker for susceptibility to tobacco-related cancers: a review of molecular epidemiological studies. Mol Carcinog. 2005; 42: 65-92. 
3 Goode EL, Ulrich CM, Potter JD. Polymorphisms in DNA repair genes and associations with cancer risk. Cancer Epidemiol Biomarkers Prev 2002; 11: 1513-1530.

4 Rajavashisth TB, $X_{u} X P$, Jovinge $S$, Meisel $S, X_{u} X O$, Chai NN, Fishbein MC, Kaul $S$, Cercek B, Sharifi B, Shah PK. Membrane type 1 matrix metalloproteinase expression in human atherosclerotic plaques: evidence for activation by proinflammatory mediators. Circulation 1999; 99: 3103-3109.

5 Androulakis E, Tousoulis D, Papageorgiou N, Latsios G, Siasos G, Stefanadis C. The role of matrix metalloproteinases in essential hypertension. Curr Top Med Chem 2012; 12: 1149-1158.

6 Schmid-Schonbein GW. Matrix metalloproteinases activities in hypertension: emerging opportunities. Hypertension 2011; 57: 24-25.

7 Back M, Ketelhuth DF, Agewall S. Matrix metalloproteinases in atherothrombosis. Prog Cardiovasc Dis 2010; 52: 410-428.

8 Jones CB, Sane DC, Herrington DM. Matrix metalloproteinases: a review of their structure and role in acute coronary syndrome. Cardiovasc Res 2003; 59: 812-823.

9 Yan C, Boyd DD. Regulation of matrix metalloproteinase gene expression. J Cell Physiol 2007; 211: 19-26.

10 Garneau NL, Wilusz J, Wilusz CJ. The highways and byways of mRNA decay. Nat Rev Mol Cell Biol 2007; 8: 113-126.

11 Kennedy AM, Inada M, Krane SM, Christie PT, Harding B, López-Otín C, Sánchez LM, Pannett AA, Dearlove A, Hartley C, Byrne MH, Reed AA, Nesbit MA, Whyte MP, Thakker RV. MMP13 mutation causes spondyloepimetaphyseal dysplasia, Missouri type (SEMD(MO). J Clin Invest 2005; 115: 2832-2842.

12 Niu W, Qi Y. Matrix metalloproteinase family gene polymorphisms and risk for coronary artery disease: systematic review and meta-analysis. Heart 2012; 98: 1483-1491.

13 Shao Y, Deng M, Lian Z, Shi M, Jiang X. [Matrix metalloproteinase-3 genotype distribution and its relation with blood pressure profiles in Guangzhou rural population]. Nan Fang Yi Ke Da Xue Xue Bao 2013; 33: 586-589.

14 Perez-Hernandez N, Vargas-Alarcon G, Martinez-Rodriguez N, Martínez-Ríos MA, Peña-Duque MA, Peña-Díaz Ade L, Valente-Acosta B, Posadas-Romero C, Medina A, Rodríguez-Pérez JM. The matrix metalloproteinase 2-1575 gene polymorphism is associated with the risk of developing myocardial infarction in Mexican patients. J Atheroscler Thromb 2012; 19: 718-727.

15 Palei AC, Sandrim VC, Amaral LM, Machado JS, Cavalli RC, Lacchini R, Duarte G, Tanus-Santos JE. Effects of matrix metalloproteinase (MMP)-2 polymorphisms on responsiveness to antihypertensive therapy of women with hypertensive disorders of pregnancy. Basic Clin Pharmacol Toxicol 2012; 111: 262-267.

16 Rizzi E, Castro MM, Fernandes K, Barbosa F Jr., Arisi GM, Garcia-Cairasco N, Bendhack LM, Tanus-Santos JE, Gerlach RF. Evidence of early involvement of matrix metalloproteinase-2 in lead-induced hypertension. Arch Toxicol 2009; 83: 439-449.

17 Qi Y, Zhao H, Wang Y, Wang Y, Lu C, Xiao Y, Cao J, Jia N, Wang B, Niu W. Replication of the top 10 most significant polymorphisms from a large blood pressure genome-wide association study of northeastern Han Chinese East Asians. Hypertens Res 2013; 37: $134-138$.

18 Liu R, Zhao H, Wang Y, Wang Y, Lu C, Xiao Y, Jia N, Wang B, Niu W. The contributory role of angiotensin receptor-like 1 gene multiple polymorphisms in hypertension among northeastern Han Chinese. PLOS ONE 2014. 9. e86095.
19 Wang Y-L, Qi Y, Bai J-N, Qi Z-M, Li J-R, Zhao H-Y, Wang Y-F, Lu C-Z, Xiao Y, Jia N, Wang B, Niu WQ. Tag polymorphisms of solute carrier family 12 member 3 gene modify the risk of hypertension in northeastern Han Chinese. J Hum Hypertens 2014; 28 : 504-509.

20 Tobin MD, Sheehan NA, Scurrah KJ, Burton PR. Adjusting for treatment effects in studies of quantitative traits: antihypertensive therapy and systolic blood pressure. Stat Med 2005; 24: 2911-2935.

21 Khanna M, Park P, Zirvi M, Cao W, Picon A, Day J, Paty P, Barany F. Multiplex PCR/LDR for detection of K-ras mutations in primary colon tumors. Oncogene 1999; 18: 27-38.

22 Pattin KA, White BC, Barney N, Gui J, Nelson HH, Kelsey KT, Andrew AS, Karagas MR, Moore $\mathrm{JH}$. A computationally efficient hypothesis testing method for epistasis analysis using multifactor dimensionality reduction. Genet Epidemiol 2009; 33: 87-94.

23 Hahn LW, Ritchie MD, Moore JH. Multifactor dimensionality reduction software for detecting gene-gene and gene-environment interactions. Bioinformatics 2003; 19 . 376-382.

24 Bashey RI, Cox R, McCann J, Jimenez SA. Changes in collagen biosynthesis, types, and mechanics of aorta in hypertensive rats. J Lab Clin Med 1989; 113: 604-611.

25 Pushpakumar S, Kundu S, Pryor T, Givvimani S, Lederer E, Tyagi SC, Sen U. Angiotensin-II induced hypertension and renovascular remodelling in tissue inhibitor of metalloproteinase 2 knockout mice. J Hypertens 2013; 31: 2270-2281; discussion 2281.

26 Qi Y, Niu W, Zhu T, Zhou W, Quu C. Synergistic effect of the genetic polymorphisms of the renin-angiotensin-aldosterone system on high-altitude pulmonary edema: a study from Qinghai-Tibet altitude. Eur J Epidemiol 2008; 23: 143-152.

27 Vlachopoulos C, Aznaouridis K, Dima I, loakeimidis N, Vasiliadou C, Zervoudaki A, Gialernios T, Stefanadis C. Negative association between serum levels of matrix metalloproteinases-2 and -9 and aortic stiffness in healthy adults. Int J Cardiol 2007; 122: $232-238$.

28 Flamant M, Placier S, Dubroca C, Esposito B, Lopes I, Chatziantoniou C, Tedgui A, Dussaule JC, Lehoux S. Role of matrix metalloproteinases in early hypertensive vascular remodeling. Hypertension 2007; 50: 212-218.

29 Kato N. Ethnic differences in genetic predisposition to hypertension. Hypertens Res 2012; 35: 574-581.

30 Pineda-Krch M, Lehtila K. Costs and benefits of genetic heterogeneity within organisms. J Evol Biol 2004; 17: 1167-1177.

31 Niu W, Qi Y. Association of alpha-adducin and G-protein beta3 genetic polymorphisms with hypertension: a meta-analysis of Chinese populations. PLOS ONE 2011; 6: e17052.

32 Cardon LR, Bell JI. Association study designs for complex diseases. Nat Rev Genet 2001; 2: 91-99

33 Gu M, Dong X, Zhang X, Wang X, Qi Y, Yu J, Niu W. Strong association between two polymorphisms on $15 q 25.1$ and lung cancer risk: a meta-analysis. PLOS ONE 2012; 7: e37970.

34 Moore JH, Ritchie MD. STUDENTJAMA. The challenges of whole-genome approaches to common diseases. JAMA 2004; 291: 1642-1643.

35 Gui J, Andrew AS, Andrews P, Nelson HM, Kelsey KT, Karagas MR, Moore JH. A simple and computationally efficient sampling approach to covariate adjustment for multifactor dimensionality reduction analysis of epistasis. Hum Hered 2010; 70: 219-225. 